\title{
La división del aire en los Quesiti et inventione diverse (1546) de Niccolò Tartaglia
}

\author{
Virginia lommi Echeverría (*) \\ (*) Instituto de Historia, Pontificia Universidad Católica de Valparaíso. \\ Viña del Mar, Chile. virginia.iommi@ucv.cl
}

Dynamis

[0211-9536] 2010; 30: 197-212
Fecha de recepción: 17 de diciembre de 2009

Fecha de aceptación: 22 de febrero de 2010

SUMARIO: 1.- Introducción. 2.-La idea de «rompimiento del aire». 3.-La fractura del aire en Vannoccio Biringuccio. 4.-Jordanus Nemorarius y la descripción de la resistencia. 5.-Filopón y la materialidad del medio. 6.-El destino de una idea.

RESUMEN: En sus Quesiti et inventione diverse (Venecia, 1546) Niccolò Tartaglia propuso una teoría del lanzamiento de proyectiles centrada en la resistencia opuesta por el aire y su subsiguiente división. Pienso que su explicación está inspirada en tres referentes, que aquí se analizan. El primero de ellos es su contemporáneo Vannoccio Biringuccio, quien formuló el concepto del fractura del aire para justificar el estruendo producido por la salida de la bala del cañón. En segundo lugar, se estudia la afinidad existente entre los Quesiti y la idea de resistencia del medio contenida en el tratado medieval De ratione ponderis, atribuido al matemático Jordanus Nemorarius (siglo XIII). Finalmente se sugiere la consonancia de la exposición tartagliana con los postulados de Juan Filopón (siglo VI) en lo referente a la materialidad del aire y su consecuente perturbación tras el lanzamiento del proyectil.

PALABRAS CLAVE: Niccolò Tartaglia, aire, balística, resistencia, dinámica, Vannoccio Biringuccio, Jordanus Nemorarius, Juan Filopón.

KEY WORDS: Niccolò Tartaglia, air, ballistics, resistance, dynamics, Vannoccio Biringuccio, Jordanus Nemorarius, John Philiponus.

\section{Introducción $(*)$}

En el Instituto y Museo de Historia de la Ciencia de Florencia se conserva una edición de 1554 de los Quesiti et inventione diverse del matemático

(*) La investigación base de este artículo fue financiada por la Università degli Studi di Firenze, el Istituto Italiano di Scienze Umane y la Pontificia Universidad Católica de Valparaíso: 
italiano Niccoló Tartaglia (c. 1500-1557) cuyas anotaciones marginales han sido atribuidas a Galileo Galilei ${ }^{1}$. A simple vista resalta el notorio interés del científico por los pasajes referidos a problemas de dinámica pues los comentarios más extensos fueron escritos al costado de estas cuestiones. En ellos se aprecia la perplejidad con la que el físico pisano alude a la descripción del modo en que los proyectiles atraviesan el medio tras ser expulsados del cañón. Galileo cuestiona las consecuencias físicas de la explicación ofrecida ya que conducía a aceptar que el «vacío es permitido en la naturaleza» ${ }^{2}$.

Tartaglia no sostenía este efecto de manera explícita, pero un lector atento como Galileo percibió que la exposición implicaba la conservación de una separación en el aire tras su penetración. El rechazo de la teoría contenida en los Quesiti es confirmada por la postilla escrita junto a un pasaje que reitera la descripción del fenómeno, en la cual señala que «esta opinión debe ser considerada con sumo cuidado» ${ }^{3}$.

En el presente artículo se analizan en detalle los tres fragmentos que concentran las críticas galileanas proponiéndose una explicación del origen y configuración de las conjeturas físicas de Tartaglia.

\section{La idea de «rompimiento del aire»}

Los estudios de balística de Niccolò Tartaglia representan una de las etapas más importantes e influyentes en la historia de la dinámica. En 1537 publicó la Nova scientia, obra destinada a inaugurar el estudio exclusivamente geométrico de la trayectoria de los proyectiles, obviando las digresiones filosóficas que habían caracterizado ese tipo de tratados hasta entonces. Su enfoque se sostenía en la exclusión de las condiciones ambientales como

1. Berti, Domenico. Storia dei manoscritti Galileiani della Biblioteca Nazionale di Firenze ed indicazione di parecchi libri e codici postillati da Galileo. Atti della Reale Accademia dei Lincei, 1875-1876; 273, serie Seconda, vol. III, parte III: 96-105 (p. 100). Para la identificación de la copia galileana con el ejemplar florentino véase Favaro, Antonio. La librería di Galileo. Miscellanea Galileiana Inedita. Reale Istituto Veneto di Scienze, Lettere ed Arti. 1882; 22: 982-1034.

2. «(...) si da vacuo nella natura». Tartaglia, Niccolò. Quesiti et inventione diverse. Venecia: Nicolo de Bescarini; 1554, f. 12v. Copia conservada en el Istituto e Museo di Storia della Scienza de Florencia. La nota corresponde a un comentario manuscrito de Galileo al problema tratado en la cuestión IV del Libro I.

3. «E da considerala bene ques'opinione». Tartaglia, n. 2, f. 33r. Galileo se refiere a la cuestión VII del Libro II. 
factor determinante, en particular de la resistencia del aire como causa de las variaciones en la velocidad y distancia alcanzadas por el objeto lanzado. Tartaglia afirmaba que en el momento inicial de la eyección, el aire era dividido por la bala de cañón dejando que ésta prosiguiese su recorrido sin perturbaciones.

Su segundo tratado, los Quesiti et inventione diverse de 1546 - recolección de diálogos que combinan especulación matemática con temas mecánicos y militares - fue un intento de respuesta a las preguntas surgidas por la aparición de su primera obra. En él se presentaba una renovada perspectiva del aire, otorgándole una importancia central en la interpretación de los fenómenos.

La cuarta pregunta del primer libro de los Quesiti proviene de la observación práctica de uno de los protagonistas del enfrentamiento entre la Orden de Caballeros de San Juan y el Imperio Otomano por la isla de Rodas en 1522, el Prior de Barletta Gabriele Tadino (c. 1475-1543) ${ }^{4}$ :

«Prior: Si disparo dos balas de cañón, una después de la otra, desde la misma elevación y dirigidas hacia el mismo lugar, quisiera saber si estos tiros serán iguales.

Niccolò: Serán diferentes sin lugar a dudas, pues el segundo llegará más lejos que el primero.

P: ¿Por qué?

$\mathrm{N}$ : Por dos razones. En primer lugar porque en el primer tiro la bala de cañón encontrará el aire quieto, mientras en el segundo lo encontrará sacudido por el primer tiro, discurriendo en dirección hacia el lugar de la caída. Es más simple mover y penetrar algo en movimiento y ya atravesado que algo tranquilo y asentado. La bala de cañón que es disparada después - debido a que encuentra menos obstáculos en su movimiento- llegará más lejos que aquella disparada inicialmente» ${ }^{5}$.

4. Masotti, Arnaldo. Gabriele Tadino e Niccolo Tartaglia. Atti dell'Ateneo di Scienze, Lettere ed Arti di Bergamo. 1973-1974; 38: 363-374. Masotti afirma que, aunque algunos autores duden de la veracidad del diálogo, no hay evidencia para desconfiar del relato de Tartaglia. Entre esos autores Masotti menciona a Giovanni Francesco Gambara, Baldassare Boncompagni, Antonio Favaro y Carlo Promis.

5. Tartaglia, Niccolò. Quesiti et inventione diverse. Venecia: Venturino Ruffinelli; 1546, f. 13r. La segunda razón, que aquí no citamos, es la diferencia entre la pólvora húmeda del primer tiro y la seca del segundo. La traducción del texto es nuestra. 
Tartaglia afronta en este fragmento un hecho que no tiene explicación en el sistema matemático que había elaborado años antes. Si la disminución de la importancia de la resistencia del aire lo llevó a una geometrización de la trayectoria de los proyectiles, el fenómeno presentado por Tadino le obliga a reconsiderar la relevancia del medio. Si la elevación y dirección del cañón explicaban las modificaciones de la trayectoria en la Nova scientia, la situación descrita por el Prior descartaba estas variables desde un principio.

En la pregunta siguiente la penetración del aire es denominada rompimento dell'aere ${ }^{6}$. Ello muestra que Tartaglia no abandona la idea de separación del medio presentada en su primer tratado y se concentra en las alternativas de penetración que pueden ser identificadas a partir de las condiciones del aire. Al clarificarlas resalta una variable no matemática de su sistema, acomodando su construcción teórica anterior a observaciones empíricas.

Veamos otro ejemplo del rol físico dado al aire. En el segundo libro, cuestión siete, Tadino vuelve a preguntar:

«P: Cuando estuve en Rodas, en la época del asedio turco, fui a un determinado lugar con algunos zapadores para hacer arreglos. Ocurrió que los turcos arrojaron una bala de cañón cuyo silbido fue escuchado por todos nosotros desde lejos, lo cual permitió que la evitásemos con facilidad. Luego del impacto, todos volvimos al trabajo confiados en que el sonido o silbido del proyectil nos advertiría. Pero cuando una segunda bala de cañón fue disparada, ésta vino con tal silencio que antes de escucharla ya había matado a cuatro zapadores. Quisiera saber por qué vino tan silenciosamente, tal como lo hicieron aquellas que la sucedieron.

$\mathrm{N}$ : Esto ocurre por las mismas razones mencionadas en la cuarta cuestión del primer libro, a saber, porque en el primer tiro la bala encuentra el aire quieto, el cual ofrece mayor resistencia a su movimiento que el aire sacudido. Pues la resistencia es la causa del silbido, que se produce por la dificultad que encuentra la bala al penetrar el aire quieto y asentado. Pero en el segundo tiro ésta halla el aire movido, roto y sacudido por la primera bala; y el aire, estando dirigido hacia el lugar de la caída, la ayuda en su movimiento. La bala, al no encontrar obstáculos en el segundo caso, no silba tan fuerte; tal como ocurre en los tiros sucesivos» ${ }^{7}$.

6. Tartaglia, n. 5, f. 13v.

7. Tartaglia, n. 5, f. 35v-36r. 
Como afirma el propio Tartaglia, se trata de otra consecuencia del mismo caso analizado antes. Esta vez no enfatiza el efecto del proyectil, sino las diferentes maneras en que un objeto afronta la presencia del aire ${ }^{8}$. El medio quieto resiste la introducción de un cuerpo ajeno y el resultado perceptible de esta lucha entre ambas fuerzas es el silbido.

El choque entre el proyectil y el aire es descrito con mayor detalle en la cuestión XVIII del primer libro de los Quesiti. Tartaglia, respondiendo a la pregunta planteada por Iacomo de Acaya - de quien sólo indica visitó Venecia para la fiesta de la Sensa en 1542- explica por qué un tiro efectuado a corta distancia tiene un efecto menor que otro realizado desde más lejos:

«S. Iacomo: Digo por experiencia que cuando una bala es lanzada con un cañón hacia un muro muy cercano, ésta no tiene efecto tan grande como cuando es lanzada desde más lejos. Por las razones aducidas en su Nova scientia debería ocurrir lo contrario, porque la bala disminuye su velocidad al alejarse del cañón. Es decir, tiene un efecto menor en el objetivo al ir más lento, de modo tal que mientras más cerca se encuentre éste del punto del disparo, mayor debería ser el efecto de la bala en el lugar del impacto, pues viaja con mayor velocidad (...). Sin embargo, empíricamente he apreciado lo contrario y quisiera saber la causa de este fenómeno.

N: Para responder a esta pregunta debe enfatizarse que todo aquel que se mueve, mueve a su vez algo más. Cuando la bala es movida por el viento causado por la pólvora, la misma bala y viento mueven en el mismo instante el aire contiguo dentro del cañón; ese aire mueve a otro junto a él y ese otro a otro, hasta que la bala empuja delante de sí una gran cantidad de aire (...). Esta penetración no continúa por mucho, pues tal figura aérea por un breve espacio de tiempo va delante de la bala, pero como ésta es de un material pesado que atraviesa con mayor facilidad el aire que la figura aérea y además va más rápido que ella, termina por dejarla atrás muy rápidamente. Volviendo a nuestro problema inicial, cuando disparas con un cañón desde muy cerca, la figura aérea que es empujada delante de la bala colisionará antes que ella. Dado que la figura no es capaz de penetrar el objetivo, es necesario que la parte más extrema de ésta reflecte y vuelva hacia el resto de la figura y la bala que le sigue (...) generando un gran contraste. (...) Pero cuando el objetivo está situado a una distancia intermedia, la bala, debido a su velocidad, deja atrás la figura aérea - si no toda, al menos parte - y tiene un efecto mayor que

8. El effetto o efecto es la ofensa o agujero causado por la bala. 
cuando está demasiado cerca, porque la bala no encuentra un impedimento en la reflexión (...) del aire.

S. Iacomo: Esta razón me parece correcta, y entiendo que el fenómeno no puede ser causado por otra cosa» ${ }^{9}$.

En este fragmento, Tartaglia intenta explicar un fenómeno que parece negar la afirmación contenida en la Nova scientia sobre la continua disminución de la velocidad en el movimiento ascendente de la bala ${ }^{10}$. Para ello se centra en el momento inmediatamente anterior al rompimiento del medio; por un breve instante, el proyectil empuja el aire que encuentra frente a él antes de penetrarlo. Para Tartaglia esta figura aerea reduce el efecto cuando el impacto es demasiado cercano ${ }^{11}$.

Alexandre Koyré interpretó este pasaje como un claro ejemplo de las dificultades que encontró Tartaglia en esta segunda etapa de su reflexión balística. Desde su perspectiva, el matemático no pudo utilizar el mismo argumento de la variación entre dos lanzamientos consecutivos, porque la modificación de la fuerza de percusión en relación al lugar de impacto era perceptible con un único tiro. Así Tartaglia se vio forzado a imaginar otra explicación y llegó a señalar paradójicamente que el aumento en la fuerza del impacto era perfectamente compatible con la disminución de la velocidad del proyectil ${ }^{12}$. No conozco otro análisis semejante en la tradición física referido a la variación en la fuerza de colisión como consecuencia de una figura aerea.

La creación de esta almohada de aire implica la formación, al menos por un instante, de un espacio vacío tras el proyectil. Si el medio desplazado se acumula frente a la bala de cañón formando una columna de aire coherente, es necesario que se concentre delante del proyectil y que, al menos inicialmente, no se mueva hacia su parte posterior.

9. Tartaglia, n. 5, f. 24v-r.

10. Tartaglia, Niccolò. Nova scientia. Venecia: Stefano da Sabbio; 1537, Libro I, Proposición III, páginas no numeradas.

11. Como reparó Gerhard Arend la gravità atribuida al aire en este caso no debe ser interpretada como el peso del elemento, sino como una habilidad que recibe en determinadas circunstancias. Véase Arend, Gerhard. Die Mechanik des Niccolò Tartaglia im Kontext der zeitgenössischen Erkenntnis-und Wissenschaftstheorie, Munich: Institüt für Geschichte der Naturwissenschaften; 1998, p. 207-208.

12. Koyré, Alexandre. La dynamique de Nicolo Tartaglia. In: Études d'histoire de la pensée scientifique. París: Presses Universitaires de France; 1966, p. 101-121 (p. 119-120). 
En la sección siguiente de la cuestión XVIII, Tartaglia muestra cuán sólida puede llegar a ser esta figura de aire. Dado que la bala no tiene tiempo de penetrarla, la columna de aire encuentra primero el lugar del impacto, rebotando e impidiendo un efecto mayor del choque del proyectil.

La noción de aire en los Quesiti parece así resolver situaciones que resultaban inexplicables en su sistema dinámico previo. Pese a la peculiaridad y sofisticación de las soluciones expuestas, el enfoque de Tartaglia no es del todo original y parece estar inspirado en lecturas realizadas algunos años antes de la publicación de su segunda obra.

\section{La fractura del aire en Vannoccio Biringuccio}

El mismo año que apareció la Nova scientia, Vannoccio Biringuccio (14801537) moría de manera sorpresiva. Se trata del autor de De la pirotechnia, uno de los más importantes e influyentes tratados sobre minerales y metales escrito en la Italia renacentista. La obra fue redactada entre 1530 y 1535 , y publicada póstumamente en $1540{ }^{13}$. Esta primera impresión se realizó en los talleres venecianos de quien fuera el editor del primer escrito de Tartaglia, Venturino Ruffinelli.

En De la pirotechnia hay una descripción de la división del aire muy similar a la de Tartaglia, aunque menos detallada que ésta ${ }^{14}$. Es notable que se encuentre inserta en el mismo contexto de la alusión tartagliana, es decir, la explicación de la producción de sonido tras un disparo. Biringuccio señala que algunos parecen sorprenderse por el estremecedor estruendo que produce la salida de la bala del cañón y se propone explicarlo en términos físicos. La principal causa de este sonido radica en que el aire exterior, que tiene su estructura unida y resistente por naturaleza, es además frío y especialmente denso. Este aire es dividido con gran violencia por el fuego y la bola que salen del cañón, generándose el perturbador sonido ${ }^{15}$.

13. Véase la introducción de Adriano Carugo a Biringuccio, Vannoccio. De la pirotechnia. Milán: Edizioni il Polifilo; 1977, p. XIV.

14. Junto a ello, Gerhard Arend ha destacado la similitud entre las descripciones de la elevación del cañón entre ambos autores, confirmando la familiaridad del autor de Brescia con este tratado. Arend, n. 11, p. 59.

15. Biringuccio, n. 13, f. 152v-153r. 
Esta descripción tiene numerosas semejanzas con los fragmentos de Tartaglia. Por una parte, se refiere de manera explícita al rompimiento del aire como la causa del estruendo. Asimismo, define el aire externo como una resistencia que se opone a la penetración de la bala expelida por el cañón. Además, enfatiza la violencia de la separación del medio y el contraste entre dos fuerzas que por un breve instante parecieran luchar. Todos estos elementos demuestran la clara proximidad entre los conceptos de rompimento dell aere de Tartaglia y de fractura dell aere utilizado por Biringuccio. No es difícil imaginar que el matemático se sintiese atraído por un análisis práctico del problema como el contenido en De la pirotechnia y que adoptase algunas de las concepciones allí descritas, puesto que al redactar sus Quesiti se proponía resolver problemas revelados por la experiencia cotidiana de los bombarderos.

La cercanía entre ambas obras se reduce a las descripciones parcialmente coincidentes de algunos fenómenos de balística. No obstante la afinidad terminológica, no hallamos en De la pirotechnia una exposición del comportamiento del aire concordante. La explicación del matemático centrada en la dificultad de penetración y en la alteración producida en el medio tras la inserción del proyectil se vincula con un texto de carácter diverso que había sido escrito al menos tres siglos antes.

\section{Jordanus Nemorarius y la descripción de la resistencia}

El tratado De ratione ponderis fue estudiado en profundidad por Pierre Duhem, quien enfatizó su importancia en la ciencia italiana del Renacimiento. Según él, el primero de los cuatro libros que lo componen había sido escrito durante la Edad Media por un discípulo del conocido matemático del siglo XIII Jordanus Nemorarius, mientras los tres restantes eran vestigios de ciencia griega probablemente transmitida a través de los árabes ${ }^{16}$. En oposición a esta interpretación, Ernst Moody argumentó que la conjetura de Duhem iba contra toda evidencia externa,

16. Duhem, Pierre. Les origines de la statique. París: Hermann; 1905, vol. I, p. 136; vol. II, p. 319. Duhem llegó a esta deducción considerando particularmente el cuarto libro, pues no le parecía que un autor del siglo XIII pudiese llegar a esas conclusiones. Véase Duhem, Pierre. Études sur Léonard de Vinci. París: Editions des archives contemporaines; 1984, vol. I, p. 316. Duhem propuso llamar al autor de la obra «Precursor de Simon Stevin y Descartes». 
pues los manuscritos identificados hasta ese momento, al igual que un testimonio casi contemporáneo, atribuían unánimemente el tratado al propio Jordanus ${ }^{17}$.

Más allá de los problemas de atribución, durante el siglo XVI el texto era considerado una obra genuina de Jordanus Nemorarius. Stillman Drake ha señalado que Tartaglia la conoció gracias al embajador español Diego Hurtado de Mendoza, quien permaneció en Venecia desde 1539 hasta 1546. Éste poseía un manuscrito que contenía no sólo el tratado De ratione ponderis, sino también una copia de la traducción por Guillermo de Moerbeke de los tratados matematicos de Arquímides, que Tartaglia usaría para su edición de $1543^{18}$. El matemático no sólo hizo una transcripción de la obra de Jordanus, sino que elaboró algunas figuras para explicar sus postulados. En 1565 el impresor veneciano Curzio Troiano publicó la primera edición de este tratado a partir de los apuntes dejados por Tartaglia tras su muerte ${ }^{19}$.

El tercer postulado del cuarto libro del tratado De ratione ponderis explica cómo un cuerpo rompe un medio resistente y cohesionado:

«Mientras más cohesionado sea el cuerpo, más resistirá. Sea el cuerpo resistente $\mathrm{ABC}$, y el objeto descendente $\mathrm{T}$, el cual en su caída golpea $\mathrm{B}$. Para que $\mathrm{T}$ pueda atravesar $\mathrm{AB}$, debe ser separado de $\mathrm{BC}$. Por consiguiente,

17. Moody, Ernst; Clagett, Marshall. The Medieval science of weights. Treatises ascribed to Euclid, Archimedes, Thabit ibn Qurra, Jordanus de Nemore and Blasius of Parma. Madison: The University of Wisconsin Press; 1960, p. 171-172. El testimonio corresponde a Richard Fournival (1201-c.1260).

18. Drake, Stillman; Drabkin, Israel E. Mechanics in Sixteeenth-century Italy. Selections from Tartaglia, Benedetti, Guido Ubaldo, and Galileo. Madison: The University of Wisconsin Press; 1969, p. 23. El manuscrito: Madrid, Biblioteca Nacional, 9119, de finales del siglo XV, contiene el Liber de ratione ponderis de Jordanus entre los f. 363v y 369r. Véase Clagett, Marshall. Archimedes in the Middle Ages. Philadelphia: The American Philosophical Society; 1964-1984, vol. 3, p. 69-71.

19. Aunque esta copia no aparece en el inventario realizado por el notario público veneciano Rocco de Benedetti en 1557, el matemático donó a Troiano todos sus libros y, cuando De ratione ponderis fue publicado, fue claramente declarado que el texto provenía de los fragmentos dejados por Tartaglia. Véase Tonni-Bozza, V. Frammenti di nuove ricerche intorno a Niccolò Tartaglia. Atti del Congresso Internazionale di Scienze Storiche. Roma: Tipografia della R. Accademia dei Lincei; 1904, vol. XII, p. 293-307; Boncompagni, B. Intorno ad un testamento inedito di Nicolò Tartaglia. In: Cremona, Luigi; Beltrami, Eugenio, eds. Collectanea mathematica in memoriam Dominici Chelini. Milán: Hoepli; 1881, p. 363-412; Jordanus Nemorarius, Iordani opusculum de ponderositate Nicolai Tartaleae studio correctum, novisque figuris auctum. Venecia: Curtius Troianus; 1565, f. 2v. 
mientras éstos sean más cohesionados, sostendrán a T inmóvil por un mayor tiempo antes de separarse, o bien serán acarreados por T en su movimiento, manteniéndose unidos por más tiempo y ofreciendo por lo tanto mayor resistencia y soporte» 20 .

Como destaca Moody, Jordanus aparentemente asimilaba la resistencia ofrecida por el medio al caso de un cuerpo rígido roto por el peso de un objeto que cae sobre él, presentando la rasgadura del medio como análoga a la ruptura o estiramiento de una vara sostenida en los extremos ${ }^{21}$. Tartaglia y Jordanus coinciden así en la concepción material del rompimiento del aire.

Es interesante destacar que el matemático renacentista ilustró este postulado con una figura conservada gracias a la reproducción hecha por Troiano (Figura 1). En su ilustración representó los dos momentos mencionados en el texto: la extensión del medio y la penetración del cuerpo en él. Para esto dibujó un ángulo, ATC, que simboliza el empuje del cuerpo y el desplazamiento parcial del medio opositor (B); y una línea horizontal, ATC, en la cual la sección B ha sido reemplazada por el cuerpo que separó el medio en dos secciones ${ }^{22}$.

El diagrama podría ser también usado para explicar la teoría del desplazamiento del aire en los Quesiti. Primero el medio se resiste a ser dividido por el cuerpo en movimiento dada su coherencia y quietud; sin embargo, tras ser separado, facilita la inserción de un cuerpo extraño. Por lo tanto, la inmediata penetración de un segundo cuerpo no sólo se vería facilitada al romperse la cohesión, sino que el mismo medio conduciría el cuerpo a través de la huella trazada con anterioridad. En su representación del segundo momento, Tartaglia no ubica el punto B, indicando que ha sido empujado hacia uno de los costados. Tal como ocurre con una vara

\footnotetext{
20. Moody y Clagett, n. 17, p. 214. La traducción es nuestra.

21. Moody y Clagett, n. 17, p. 407.

22. Es importante destacar que el diagrama contenido en la edición de Moody, sólo ilustra el momento inicial sin considerar la separación del medio. En esta figura, basada en las ilustraciones encontradas en los manuscritos medievales, el cuerpo en movimiento, T, empuja el medio hacia abajo. Interpreto el «sic aut» escrito por Tartaglia en su ilustración, no como una distinción entre el medio cohesionado que impide el movimiento de T y la cesión del medio a este último, sino como la diferenciación entre penetración y extensión o alargamiento, puesto que B no es incluido en la línea horizontal.
} 
$a$

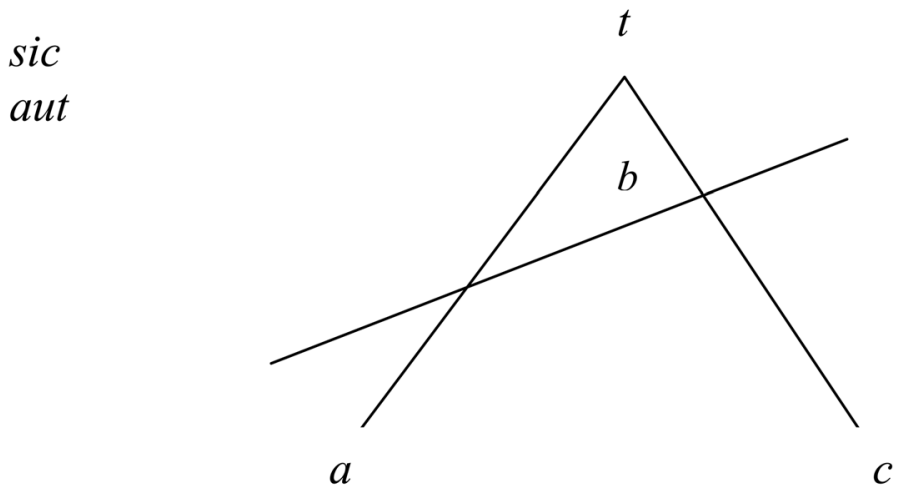

Figura 1. Reproducción de la figura contenida en Niccolò Tartaglia. Iordani opusculum de ponderositate. Venecia: apud Curtius Troianum; 1565, fol. 13v.

que es quebrada en dos secciones, todas sus partes se separan entre las porciones restantes.

La interpretación de este postulado revelada en la figura de Tartaglia, propone también un interesante giro: el cuerpo T se mueve hacia arriba, indicando que el proceso descrito en De ratione ponderis IV, 3, puede ser aplicado al movimiento ascendente. Este aspecto intrascendente en términos geométricos tiene una gran relevancia física.

Según Duhem, Tartaglia habría admitido que un proyectil aceleraría su velocidad de caída gracias a la acción del aire. El historiador francés afirmó que podía reconstruirse una importante tradición considerando las diferentes teorías formuladas en torno al rol de este elemento como conservador del movimiento en la caída acelerada de los cuerpos graves. Para Duhem, el texto de Jordanus constituye uno de los medios de divulgación más influyentes de la idea que el aire desplazado favorece el aumento de velocidad del proyectil ${ }^{23}$.

23. Duhem, 1984, n. 16, vol. I, p. 136-137; Duhem, 1905, n. 16, vol. I, p. 138; Duhem, Pierre. Le Système du monde. Histoire des doctrines cosmologiques de Platon à Copernic. París: Hermann; 1954-1965, vol. VIII, p. 251. Esta posibilidad habría sido sugerida por el mismo Aristóteles en De caelo III, 2. 
Duhem centró su análisis en los dos primeros fragmentos que hemos citado de los Quesiti, y los relacionó con el sexto postulado del libro cuarto del tratado De ratione ponderis. En él, Jordanus afirma que los cuerpos al caer atraviesan distintas capas de un medio, las cuales se van ubicando sucesivamente en su sección posterior, aumentando de esta manera su peso y velocidad de caída ${ }^{24}$.

Sin embargo, Tartaglia no alude al aire como propulsor en su comparación de dos disparos consecutivos. Únicamente afirma que tras ser penetrado el aire «tiende» al lugar de caída, pues los términos tendente o scorrente se refieren a una traza continua dejada por la primera bala de cañón. No he encontrado en este tratado un análisis condicionado por la idea del aire como un conservador del movimiento, sino una interpretación del modo cómo el aire cede al objeto extraño ${ }^{25}$.

Ahora bien, Jordanus parece también describir un proceso equivalente al de formación de la figura aerea en su tratado:

«Si un cuerpo cuyas partes están cohesionadas es obstruido directamente en su movimiento, éste retrocederá directamente. Esto ocurre por el medio en el cual el cuerpo es transportado, ya sea agua o aire (...). Sea el medio en el cual se mueve B, el cuerpo A y el lugar de impacto C. Dado que A mueve B, cuando A abandona su lugar y expele B del suyo, B tiene que retroceder para ocupar el espacio de atrás. Entonces [el cuerpo] es empujado hacia adelante y retrocedido a la parte posterior por el mismo impulso» ${ }^{26}$.

Si entendemos este postulado considerando el disparo de una bala, veremos que Tartaglia no sólo lo utilizó sino que también lo adaptó a su teoría física. De hecho, esa modificación revela la diferencia fundamental entre ambos autores. El autor renacentista en la cuestión XVIII no se refiere a los espacios que deben ser llenados, prefiriendo centrarse en el rol de la columna de aire y su efecto en la reducción del impacto. Esto significa que para Tartaglia cuando A expele B de su lugar, por un breve instante B no revierte su movimiento para evitar el vacío, sino que avanza hasta encontrarse con el objeto que se interpone en la trayectoria. Así B rebota primero, dado que no

\footnotetext{
24. Moody; Clagett, n. 17, p. 216-217.

25. Esto es confirmado por la reedición hecha por el autor de la Nova scientia en 1550, en la que no incluyó modificaciones a su teoría geométrica. Si en 1546 otorgó una función central al aire, fue para analizar situaciones que no podían ser comprendidas desde la abstracción.

26. Moody; Clagett, n. 17, p. 224-225.
} 
puede penetrar $\mathrm{C}$, y sólo entonces una porción del aire retrocede, afectando la fuerza y el efecto del proyectil. En otras palabras, la teoría de Tartaglia es radicalmente diferente, pero su forma y lenguaje son sorprendentemente similares a los de Jordanus en esta proposición ${ }^{27}$. Como él, basa su explicación en la interacción de $\mathrm{A}, \mathrm{B}$ y $\mathrm{C}$, pero por un instante les da funciones totalmente distintas a las que tienen en el postulado medieval.

Esta modificación del modelo jordaniano evidencia un elemento que me parece importante analizar con mayor profundidad. Tartaglia atribuye al medio una consistencia que permite imaginar un fenómeno como el de la figura aerea. Esta posibilidad enlaza su exposición con un autor bizantino que alcanzó gran popularidad durante el siglo XVI.

\section{Filopón y la materialidad del medio}

Una de las fuentes más influyentes en relación al análisis del movimiento de proyectiles se difundió precisamente durante los años que Tartaglia dedicó a trabajar en este tema. Nos referimos al comentario a la Física de Aristóteles del autor bizantino Juan Filopón (siglo VI). La importancia de esta obra durante la Edad Media no está clara porque no existen menciones explícitas a ella. Sin embargo, durante la segunda mitad del siglo XV es posible identificar una tradición manuscrita, ya que el texto aparece en los inventarios de la biblioteca del cardenal Bessarion (1402-1472), la biblioteca de San Marco en Florencia y la famosa colección privada de Giovanni Pico della Mirandola (1463-1494) ${ }^{28}$. La traducción al latín de Dorotheus fue publicada en Venecia en 1539, cuatro años después de la

27. Mario Piotti ha señalado que el concepto de riflessione entendido como la desviación del movimiento de una masa fluida determinada por el impacto con otra masa, hizo su primera aparición en textos italianos a finales del siglo XV, y que Tartaglia es el primer autor que usa riflettere para expresar el rebote contra un obstáculo, o retrahere, diríamos en lenguaje de Jordanus. Piotti, Mario. «Un puoco grossetto di loquella». La lingua di Niccolò Tartaglia. «La Nova Scientia» e i «Quesiti et inventione diverse». Milán: Ed. Universitarie di Lettere Economia Diritto; 1998, p. 224.

28. Schmitt, Charles. Philoponus' commentary on Aristotle's Physics in the Sixteenth Century. In: Sorabji, Richard, ed. Philoponus and the rejection of Aristotelian science. London: Duckworth, 1987, p. 210-230. Para la copia en Venecia ver Labowsky, Lotte. Bessarion's Library and the Biblioteca Marciana. Six early inventories. Roma: Storia e Letteratura; 1979, p. 221-222. Para la copia de San Marco, véase Ullman, Berthold L.; Stadter, Philip A. The public library of Renaissance Florence. Padua: Antenore; 1972, p. 257. Para el ejemplar de Pico della Mirandola véase 
edición griega ${ }^{29}$. El primer autor influenciado directamente por el comentario fue Gianfrancesco Pico della Mirandola (1470-1533), quien lo utilizó extensamente en su Examen vanitatis doctrinae gentium (1520) para atacar la filosofía de Aristóteles ${ }^{30}$. Siguiendo a Filopón, negaba el vínculo causal entre el medio y la producción del movimiento ${ }^{31}$. Es justamente esta idea uno de los legados más significativos del autor griego al pensamiento renacentista y, al apreciarla en detalle, se manifiesta su consonancia con el enfoque tartagliano.

Según Aristóteles, la materia de un cuerpo permanece idéntica al variar su volumen, de tal modo que, por ejemplo, la contracción o extensión del aire responde a una cualidad propia de éste, y no a la presencia de espacios vacíos. Esta teoría prevaleció por más de mil años y se sostuvo en la comúnmente aceptada definición del mundo como un continuo de materia. En el otro extremo se ubicaba la interpretación atomista, la cual reconocía únicamente la existencia de los átomos y el vacío, explicando los cambios y movimientos a partir de la interacción entre ambos. Como ha mostrado Charles B. Schmitt, estas lecturas tuvieron una renovada fortuna durante el siglo XVI ${ }^{32}$. La popularidad de Filopón debe insertarse en este contexto más amplio.

En su negación de la existencia del vacío, Aristóteles argumentaba que el movimiento violento o forzado no podría tener lugar si hubiese espacios vacantes, puesto que el medio cumple un rol esencial en la continuación del avance de los proyectiles. Filopón dedicó largos pasajes de su obra a refutar este principio y desarrolló una interesante teoría sobre la penetración del aire. Según el Estagirita, el aire que es movido en primera instancia cuando el proyectil es descargado, recibe un impulso que empuja al objeto. Este aire permanece en contacto con el cuerpo hasta que la fuerza motriz impresa en un comienzo se disipa. El autor bizantino señaló que esta explicación carecía de fundamentos dado que la experiencia demostraba que, incluso si uno agitaba aire con gran vehemencia, no conseguiría mover un objeto

Kibre, Pearl. The library of Giovanni Pico della Mirandola. Nueva York: Columbia University Press; 1936, p. 15.

29. Para una lista de las ediciones del siglo XVI de esta obra, véase Schmitt, n. 28, p. 229.

30. Schmitt, Charles. Gianfrancesco Pico della Mirandola (1469-1533) and his critique or Aristotle. La Haya: International Archives of the History of Ideas, Martinus Nighoff; 1967.

31. Schmitt, n. 28, p. 221-222.

32. Schmitt, Charles. Experimental evidence for and against void: the sixteenth-century arguments. Isis. 1967; 58: 352-366. 
de una manera equivalente a la del movimiento de un proyectil ${ }^{33}$. Junto con esto Filopón afirmaba que la explicación aristotélica era tan improbable como la principal teoría refutada en la Física: la antiperístasis o excitación recíproca de cualidades contrarias. El principal problema de esta noción radicaba según Filopón en la complejidad de los movimientos realizados por el aire, el cual primero era empujado junto al proyectil, luego retrocedía y finalmente proseguía hacia adelante. Para el comentarista era inexplicable que el aire cambiase la dirección dada por el impulso recibido ${ }^{34}$. El bizantino propuso entonces que la causa del movimiento era la fuerza impresa por el lanzador en el proyectil, señalando que nada impediría el desplazamiento de un cuerpo en el vacío - como había afirmado Aristóteles- dado que el medio no cumplía rol alguno en su avance, constituyendo más bien un impedimento.

$\mathrm{Al}$ contrastar los argumentos presentados por Filopón con la dinámica de Tartaglia surgen interesantes elementos en común. En ambos casos el medio aparece como una resistencia que es desplazada al inicio de la trayectoria por el proyectil y viaja una distancia significativa en esa dirección. Además los dos autores niegan la acción del aire como transmisor del impulso de movimiento, algo que el matemático renacentista había percibido desde su Nova scientia al eliminarla como variable en la trayectoria de las balas de cañón. Filopón, por su parte, dedica una larga sección de su exposición a describir el comportamiento del aire, y la función que le atribuye es muy similar a la que Tartaglia incorporó en 1546: se trata de un cuerpo consistente perturbado por la penetración del proyectil y trasladado por la fuerza de éste. El medio no es considerado entonces como el espacio atravesado, sino como una materia que es movida junto al objeto, rasgo que distingue a los Quesiti de la Nova scientia.

Pese a que Tartaglia no menciona a Filopón en su obra, probablemente había conocido sus ideas puesto que Hurtado de Mendoza poseía una copia del Examen vanitatis de Gianfrancesco Pico della Mirandola ${ }^{35}$.

33. Philoponus. Commentary in Aristotle's Physics, 639.3-642.9, citado en Cohen, Morris R.; Drabkin, Israel E. A source book in Greek Science. Cambridge (Mass): Harvard University Press; 1975, p. 221-222. En su ejemplo, Filopón ubicaba el objeto en la parte superior de una vara para reducir la fricción al mínimo y así permitir al aire acceder a éste casi en su totalidad, circunstancia que no alteraba su observación final.

34. Philoponus, n. 33, p. 221-222.

35. Hobson, Anthony. Renaissance book collecting: Jean Grolier and Diego Hurtado de Mendoza, their books and bindings. Cambridge: Cambridge University Press; 1999, p. 185 (ítem 861 del catálogo). 


\section{El destino de una idea}

La concepción de aire presentada por Tartaglia en sus Quesiti revela dos importantes características de su pensamiento. Por una parte, permite reconstruir el modo en que leyó e interpretó a sus predecesores. El matemático escogía referencias específicas que combinaba con su propia apreciación de las mismas, evitando repetir integralmente argumentos ajenos e insertándolos dentro de una original exposición dinámica. Por otra parte, este método muestra la peculiaridad de su enfoque, pues se desconocen obras anteriores que hayan atribuido al aire un rol en conjunto semejante. Tartaglia identificó como fenómeno central del movimiento de proyectiles la separación gradual del medio, si bien su análisis contenía ciertas ambigüedades que Galileo notaría años después con asombro.

Tartaglia no se refirió nuevamente al problema y tras la reedición en 1550 de su Nova Scientia no volvió a publicar reflexiones sobre balística. Sus últimos años los dedicó al estudio de fenómenos hidrostáticos y publicó en 1551 dos obras al respecto, la Travagaliata invenzione y los Ragionamen$t i^{36}$. En esta última se declaró explícitamente contrario a la existencia del vacío y comulgó con la idea de horror vacui ${ }^{37}$. Probablemente las críticas levantadas por su idea de división del aire fomentaron esta variación, pero hasta la fecha no hay pruebas que lo demuestren.

\section{Agradecimientos}

Quisiera agradecer a Charles Burnett, quien dirigió la tesis doctoral en la cual se basa este artículo, al Istituto e Museo di Storia della Scienza de Florencia por permitirme el acceso a la copia comentada de los Quesiti, y a los lectores anónimos y editores de esta revista por sus valiosas sugerencias.

36. Tartaglia, Niccoló. Regola generale da sulevare con ragione e misura non solamente ogni affondata nave ma una torre solida di metallo. Trovata da Nicolo Tartaglia, delle discipline mathematice amatore intitolata la travagliata inventione. Venecia: Nicolo Bescarini; 1551. Tartaglia, Niccolò. Ragionamenti sopra la sua travagliata inventione. Venecia: Nicolo Bescarini; 1551.

37. Tartaglia, n. 36, Ragionamenti, Diálogo con Richard Wentworth, Segundo Razonamiento. 